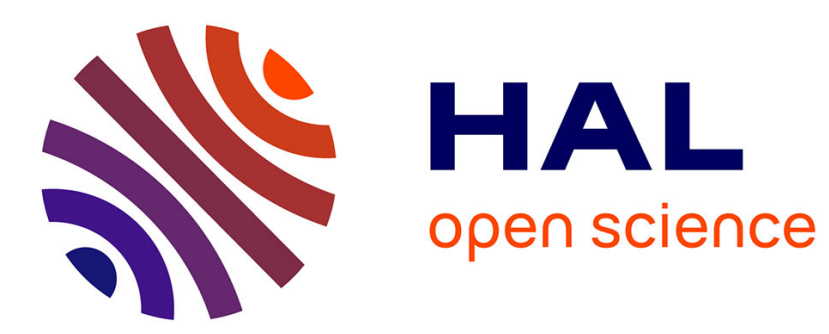

\title{
Teenagers' Stress Detection Based on Time-Sensitive Micro-blog Comment/Response Actions
}

\author{
Liang Zhao, Jia Jia, Ling Feng
}

\section{To cite this version:}

Liang Zhao, Jia Jia, Ling Feng. Teenagers' Stress Detection Based on Time-Sensitive Micro-blog Comment/Response Actions. 4th IFIP International Conference on Artificial Intelligence in Theory and Practice (AI 2015), Oct 2015, Daejeon, South Korea. pp.26-36, 10.1007/978-3-319-25261-2_3 . hal-01383941

\section{HAL Id: hal-01383941 \\ https://inria.hal.science/hal-01383941}

Submitted on 19 Oct 2016

HAL is a multi-disciplinary open access archive for the deposit and dissemination of scientific research documents, whether they are published or not. The documents may come from teaching and research institutions in France or abroad, or from public or private research centers.
L'archive ouverte pluridisciplinaire HAL, est destinée au dépôt et à la diffusion de documents scientifiques de niveau recherche, publiés ou non, émanant des établissements d'enseignement et de recherche français ou étrangers, des laboratoires publics ou privés. 


\title{
Teenagers' Stress Detection based on Time-Sensitive Micro-blog Comment/Response Actions
}

\author{
Liang Zhao, Jia Jia, and Ling Feng \\ Department of Computer Science and Technology \\ Tsinghua University, Beijing 100084, China \\ zhaoliang0415@gmail.com; \{jjia,fengling\}@tsinghua.edu.cn
}

\begin{abstract}
Accurately detecting psychological stress in time is a significant issue in the modern stressful society, especially for adolescents who are not mature enough to cope with pressure well. Micro-blog offers a new channel for teens' stress detection, since more and more teenagers nowadays prefer to express themselves on the lively virtual social networks. Previous work mainly rely on tweeting contentsto detect tweeters' phycological stress. However, a tweet is limited to 140 characters, which are too short to provide enough information to accurately figure out it$\mathrm{s}$ tweeter's stress. To overcome the limitation, this paper proposes to leverage details of social interactions between tweeters and their following friends (i.e., time-sensitive comment/response actions under a tweet) to aid stress detection. Experimental results through a real user study show that time sensitivity of comment/response acts plays a significant role in stress detection, and involving such interaction acts can improve the detection performance by $23.5 \%$ in F-measure over that without such interactions.
\end{abstract}

\section{Introduction}

The increasingly faster life pace in the competitive society often makes people stressful, especially for teenagers who are too immature to deal with psychological pressures. Currently, 20\% teenagers have psychological illness around the world [2]. An online survey of 1018 U.S. teens (aged 13-17) made by the American Psychological Association in August, 2013 found that teens suffered stress in all areas of their lives, from school to friends, work and family, which negatively affected every aspect of their lives, and about $27 \%$ of the teens experienced extreme stress and 55\% experienced moderate stress in the past school year [4]. If such pressures cannot get properly relieved in time, the teenagers will suffer severe physical and mental problems under accumulated pressures, such as clinical depressions, insomnia, and even committing suicide. According to China Center for Disease Control and Prevention [1], suicide has become the top cause of death among Chinese youth, and excessive stress is considered to be a major factor of suicide. Also in Korea, suicide has become teenagers' No.1 killer in the past two years [3]. Annual increase of adolescent suicide rate has become a world-wide 
common problem.

As adolescence is a critical period for one's growth and development, it has significant value to pay attention to teenagers' psychological status and discover their suffering pressures in time. With the popularity of social networks, micro-blog offers another low-cost sensing channel to analyze teenagers' psychological pressures through their tweets, since more and more teenagers turn to micro-blog for information acquisition, personal interaction, self-expression, and emotion release. Some research work has already made efforts to detect user's stress and depression through micro-blog by analyzing their tweeting behaviors $[13,9,8,18,15]$. Leveraging tweeting contents such as linguistic and visual features to analyze user's stress has been proven feasible $[16,11]$. However, a tweet faces the limitation of only 140 characters, which is too short to provide enough content information to figure out stress and sometimes users may not express their stress so directly. To address the limitation, we propose to further involve comment/response acts under the tweet (comments, responses, likes and forwards) to better supplement stress detection. Such interactions are attached with unique timestamps. Considering time sensitivity, we select the interactions within a certain time gap right after the tweet is posted and construct a timesensitive feature space for stress detection together with content of the tweet. Based on [16], we combine the content of both the tweet and time-sensitive comment/response acts under the tweet to extract content features for the four stress categories (academic, affection, interpersonal, self-cognition), respectively. From the observations that stressful tweets often receive more comments from friends, especially comments with care, comfort and encourage, and the psychological study [5] that users get inactive when suffering stress, we extract several novel interacting behavior features such as effective comment rate, reply rate, effective reply rate, and average interaction depth to help improve the stress detection. Our user study of 36 high school teenagers shows that time sensitivity of comment/response acts plays a significant role in stress detection and such acts in 30 minutes after the tweet posted are proved to be the most effective. Involving such interaction acts improves the detection performance by $23.5 \%$.

To the best of our knowledge, this is the first work combining tweet contents and time-sensitive comment/response acts under the tweet for stress detection. There are also no previous stress detection work, specifying features for different stress categories.

The remainder of the paper is organized as follows. We review the related work in Section 2. Section 3 analyzes and extracts features from time-sensitive comment/response acts for teens' stress detection. Experimental performance through a real user study is evaluated in Section 4. Finally, we conclude the paper in Section 5.

\section{Related Work}

Computer-aided sentiment analysis and applications in social network has drawn much attention in recent years $[16,10,11,19]$. Many studies focus on the 
single tweet, leveraging content features such as text-based linguistic attributes and visual factors such as emoticons and images. [20] proposed a system called Moodlens to do sentiment analysis for Chinese Weibo via emoticons in tweets. The emoticons are divided into four different sentiment categories (i,e, angry, disgusting, joyful and sad), and a fast Naive Bayes classifiers works for the sentiment analysis of tweets.

Beside the content features, simple social interaction attributes are also involved. [12] proposed to detect user's psychological stress from social media via a deep convolution network on sequential time series in a certain time period. Simple social features like the number of -mentions, -replies, comments and likes are considered. User-level social connections such as (mutually) follow relationships and (mutually) -mentioned relationships were also investigated in [14] to improve sentiment analysis in Twitter. [17] explored comments to help predict emotions expressed by images posted on Flickr. However, time sensitivity of such social interaction attributes is not considered in these work.

The most closest related work is [16,11]. Focusing on the four main kind$\mathrm{s}$ of stress (academic, affection, interpersonal and self-cognition) that troubles teenagers, [16] extracted different features from teenagers' tweets, such as negative emotion words, negative emoticons, unusual post time and post frequency, etc., and several classifiers were leveraged to learn the potential stress category and corresponding stress level (0-5) behind the tweet. However, [16] only considered the content and unusual posting behavior of the tweet, ignoring social interactions such as comments and replies under the tweet. Besides, the detection process only returns one stress category and one level for a single tweet, but actually a single tweet may express several kinds of stress with different levels. [11] designed a deep sparse neural network to detect stress for arbitrary micro-blog users, using content features of the tweet including linguistic attributes as [16], visual attribute like color theme, brightness, etc, and simple social attributes like number of comments, retweets and favorites. they do consider the social factors, but ignore the details such as content of comments and the interaction of friends' comments and author's reply under the tweet. They only detected whether the user suffers stress, but did not quantitatively measure the stress level. Similarly, [11] also returned one stress category for one single tweet. In addition, for both the two work, content features are not category-specified and features are all static, no time-sensitive factor considered.

\section{Stress Detection with Time-sensitive Comment/Response Acts}

Before introducing the details of stress detection, we first illustrate some notations used in the following of the paper by Table 1. And without loss of generality, we use interaction acts and comment/response acts interchangeably in the rest of the paper. Given a tweet $p=T_{w}\left(u, t_{0}\right.$, cont,$\left.S I^{\Delta t}\right)$, four kinds of interaction acts under $p$ constitute $S I^{\Delta t}$, comments, likes, and forwards from 
friends, as well as user's responses. When $\Delta t$ is big enough, then $S I^{\Delta t}$ contains all the comments, replies, likes and forwards under $p$.

As in [16], let $\mathcal{C}_{\text {ategory }}=\{$ academic, affection, interpersonal,self-cognition $\}$ be the set of teenagers' stress categories involved in the study, and $\mathcal{L}_{\text {evel }}=\{$ null, light, moderate,strong $\}$ be the set of stress levels, where null means no-stress. For a single tweet $p$, we leverage time-sensitive interaction acts $S I^{\Delta t}$ together with content of $p$ to form a feature space. Exploiting classification methods, the stress detection result of $p$ is represented as $\operatorname{Stress}(p)=\left(\left(C_{i}, L v_{1}\right), \ldots,\left(C_{4}, L v_{4}\right)\right)$, where $C_{i} \in \mathcal{C}_{\text {ategory }}$ and $L v_{i} \in \mathcal{L}_{\text {evel }}$. If $\forall L v_{i}=$ null $(\mathrm{i}=1, \ldots, 4)$, then $\operatorname{Stress}(p)$ is a zero vector and $p$ is a non-stressful tweet. Without loss of generality, we call those tweets with non-zero stress vector stressful posts.

Table 1. Notations covered in tweets

\begin{tabular}{|c|c|c|}
\hline Notation & Representation & Description \\
\hline $\mathrm{p}$ & $p=T_{w}\left(u, t_{0}\right.$, cont,$\left.S I^{\Delta t}\right)$ & $\begin{array}{l}\text { A tweet posted by user } u \text { at time } t_{0} \\
\text { with the content of cont, and set of } \\
\text { time-sensitive interactions acts } S I^{\Delta t} \\
\text { during }\left[t_{0}, t_{0}+\Delta t\right]\end{array}$ \\
\hline $\mathrm{F}$ & $F=\{f \mid f$ is a friend of $u\}$ & Friend set of user $u$ \\
\hline c & $c=\operatorname{comm}\left(f, t_{c}\right.$, cont,$\left.p\right), f \in F$ & $\begin{array}{l}\text { A comment from } f \text { at time } t_{c} \text { under } \\
\text { tweet } p \text {, and cont is the content of } \\
\text { the comment }\end{array}$ \\
\hline $\mathrm{r}$ & $r=\operatorname{rep}\left(u, t_{r}, c, \mathrm{cont}, p\right)$ & $\begin{array}{l}u \text { 's reply to comment } c \text { at time } t_{r} \\
\text { under tweet } p \text {, and cont is the } \\
\text { content of the reply }\end{array}$ \\
\hline $\mathrm{lk}$ & $l k=\operatorname{like}\left(f, t_{l}, p\right), f \in F$ & Friend $f$ puts a like seal on $p$ at $t_{l}$ \\
\hline $\mathrm{fw}$ & $f w=f w r d\left(f, t_{f}, p\right), f \in F$ & Friend $f$ forwards $p$ at time $t_{f}$ \\
\hline$C_{o m m}(f, p, \Delta t)$ & $=\left\{c \mid c . t_{c} \in\left[t_{0}, t_{0}+\Delta t\right]\right\}$ & Set of comments from $f$ within $\Delta t$ \\
\hline$R_{e p}(u, f, p, \Delta t)$ & $=\left\{r \mid r . t_{r} \in\left[t_{0}, t_{0}+\Delta t\right]\right\}$ & Set of replies to $f$ within $\Delta t$ \\
\hline$L_{i k e s}(F, p, \Delta t)$ & $=\left\{l k \mid \forall f \in F, l k . t_{l} \in\left[t_{0}, t_{0}+\Delta t\right]\right\}$ & Set of likes within $\Delta t$ \\
\hline$F_{w r d s}(F, p, \Delta t)$ & $=\left\{f w \mid \forall f \in F, f w \cdot t_{f} \in\left[t_{0}, t_{0}+\Delta t\right]\right\}$ & Set of forwards of $p$ within $\Delta t$ \\
\hline
\end{tabular}

\subsection{Modified Content Features}

A tweet is only 140 characters limited which is too short to provide enough content information to figure out stress and sometimes users are not likely to express their stress so directly via a brief tweet. Through daily observations, content of the communication between the user and his/her friends under the tweet provides powerful cues to help analyze stress. We consider the content of user interactions with friends (i.e., comments from friends and the user's replies) as part of the tweet to supplement more content information. Assume the combined content of $p$ is denoted as

$C \operatorname{Cont}(p, \Delta t)=\bigcup_{f \in F}\left\{c . c o n t, r . \operatorname{cont} \mid c \in C_{\text {omm }}(f, p, \Delta t), r \in R_{e p}(u, f, p, \Delta t)\right\} \bigcup p . c o n t$ 
Particularly, interactive comments and responses between user $u$ and friend $f$ are closely related in content and constitute a dialog. We take the content of dialog as a whole, and regard it as an item piece of $C \operatorname{Cont}(p, \Delta t)$. For each item piece in $C \operatorname{Cont}(p, \Delta t)$, we leverage a graph-based Chinese parser $[6,7]$ to analyze linguistic associations, including the correspondence between stress categories and negative emotion words, and the grammatical relation between adverbs of degree and the modified negative emotion words, etc. A single tweet may convey several kinds of stress at the same time. Thus, for each stress category in $\mathcal{C}_{\text {ategory }}$, we specify content features from $\operatorname{CCont}(p, \Delta t)$ as in [16]. i.e., number of negative emotion words, number of positive and negative emoticons, number of exclamation and question marks, emotional degree, etc.

\subsection{Time-sensitive Comment/Response Features}

Comment/Response acts under a tweet is time-sensitive especially when the user is suffering from stress. For example, when a user posts a tweet expressing depression, his/her friends will always make in-time comments within a short time gap after the tweet is released. We select the interaction acts under the tweet $p$ within $\left[t_{0}, t_{0}+\Delta t\right]$ to extract the comment/response features.

Commenting features from crowd Comments, likes and forwards are basic commenting features and always show the social attention to a tweet.

Number of comments from friends. The set of all the comments under the tweet $p$ within $\left[t_{0}, t_{0}+\Delta t\right]$ is

$$
C_{\text {omments }}(F, p, \Delta t)=\bigcup_{f \in F} C_{o m m}(f, p, \Delta t)
$$

Number of likes. People always put a like seal on a tweet to show their positive emotion or attitude to the tweet. Compared with those positive tweets, stressful tweets (negative tweets) obtain much less likes, even no like. We take the number of likes under a tweet as an indicator of potential stress, denoted as $\left|L_{i k e s}(F, p, \Delta t)\right|$.

Number of forwards. Users tend to forward public information such as news, advertisements and jokes, and seldom retweet personalized stressful tweets of another user. So stressful tweets enjoy less possibility to be forwarded by friends. The number of forwards here is denoted as $\left|F_{w r d s}(F, u, p, \Delta t)\right|$.

Number of effective comments. Among all the comments from friends, we further check the details of the comments and distinguish those containing care, comfort, or encourage (e.g., "What's up?", "Don't worry", "Everything will be OK", or hug emoticon, etc.). Such comments are considered as effective comments. The set of effective comments under tweet $p$ is denoted as $E C_{\text {omments }}(F, p, \Delta t)$. A lexicon with 132 such Chinese phrases, sentences and emoticons is constructed to identify effective comments. A tweet with more effective comments is more likely to be stressful. 
Effective comment rate (short for ECR, denoted as $\operatorname{ECR}(F, p, \Delta t)$ ) is calculated as

$$
\operatorname{ECR}(F, p, \Delta t)=\frac{\left|E C_{\text {omments }}(F, p, \Delta t)\right|}{\left|C_{\text {omments }}(F, p, \Delta t)\right|+1}
$$

where $|(\cdot)|$ stands for the number of items in set $(\cdot)$ and we smooth the equation by adding 1 upon denominator. Obviously, a higher ECR denotes a bigger probability that the tweet is stressful.

Particularly, with any two of the three features (number of comments/effective comments and effective comment rate) we can deduce the other one. Thus, for commenting features we can choose any two.

Replying features of the user Besides the social behaviors of the crowd (friends), behaviors of the user himself also make sense in stress detection. Psychological study [5] shows that a user in stress may perform low activeness in social networks. Such inactiveness can be revealed from user's reactions to the comments from friends. Three features corresponding to the user's replying behaviors are identified.

Reply rate. If a user is absorbed in big stress, s/he tends to be inactive, which can be revealed from his/her activeness of reply to the comments. The reply rate (short for $\mathrm{RR}$, denoted as $R R(u, p, \Delta t)$ ) is calculated by the rate of replies over total number of comments. Assume $R_{\text {eplies }}(u, F, p, \Delta t)=$ $\bigcup_{f \in F} R_{e p}(u, f, p, \Delta t)$ is the set of user replies to all the friends under $p$, then

$$
R R(u, p, \Delta t)=\frac{\left|R_{\text {eplies }}(u, F, p, \Delta t)\right|}{\left|C_{\text {omments }}(F, p, \Delta t)\right|+1}
$$

A lower reply rate suggests that the user is not so active and it is highly possible that $\mathrm{s} /$ he is suffering stress. Similarly, among the three features (number of comments/replies, and reply rate), we can deduce the other one with any two.

Effective reply rate. Effective replies are those responses to the effective comments. The set of effective replies under $p$ within $\left[t_{0}, t_{0}+\Delta t\right]$ is

$$
E R_{\text {eplies }}(u, F, p, \Delta t)=\left\{r \mid r . c \in E C_{\text {omments }}(F, p, \Delta t), r . t_{r} \in\left[t_{0}, t_{0}+\Delta t\right]\right\}
$$

We measure the effective reply rate (short for $\operatorname{ERR}$, denoted as $E R R(p, \Delta t)$ ) by the proportion of effective reply over the total number of effective comments.

$$
\operatorname{ERR}(p, \Delta t)=\frac{\left|E R_{\text {eplies }}(u, F, p, \Delta t)\right|}{\left|E C_{\text {omments }}(F, p, \Delta t)\right|+1}
$$

Average interaction depth with friends. A friend may comment multiple times under the same tweet, and the user may also make corresponding replies. Then, such interaction between them constitutes a dialog. In the dialog, once a new comment-reply pair comes, the interaction depth between the friend and the user accordingly increases by 1 . Assume a friend $f$ and the user $u$ make a comment-reply dialog under tweet $p$, then the interaction depth between $f$ and $u$ in tweet $p$ within $\left[t_{0}, t_{0}+\Delta t\right]$ is calculated as

$$
i D e p(f, u, p, \Delta t)=\min \left(\left|C_{\text {omm }}(f, p, \Delta t)\right|,\left|R_{e p}(u, f, p, \Delta t)\right|\right)
$$


For all the friends who comment the tweet, the average interaction depth is computed by

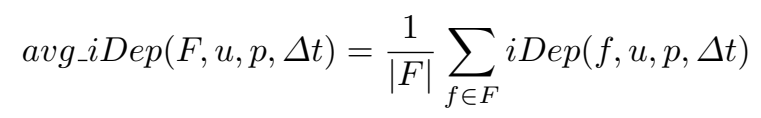

The average interaction depth reveals the user's activeness as well. The smaller the average interaction depth is, the less active the user performs, so that s/he has a higher risk of stress suffered.

\section{Experimental Study}

\subsection{Setup}

Psychological stress detection is a highly personalized issue and there are no available benchmark specially for the problem. In this work, we collect data ourselves and conduct a user study on a real micro-blog data set. 36 high school students (15 males and 21 females, aged between 15 and 17) in Shaanxi Province, China, participated in the user study. 21,648 tweets from 2013/1/1 to 2015/5/1 were collected from their accounts in Tencent Weibo ${ }^{1}$, averagely 636 tweets per teenager. Recalling the real situations, they were asked to scan their own tweets one by one and annotated the psychological stress on the four stress categories in $\mathcal{C}_{\text {ategory }}$ and corresponding stress levels in $\mathcal{L}_{\text {evel }}$ expressed in each single tweet. We take the annotation as the ground truth. For each teenager, chronologically, we use the early $66 \%$ of his/her tweets as the training data, and the rest $34 \%$ as the testing data.

\subsection{General Performance of Comment/Response acts}

Four different classifiers, including Naive Bayes, Logistic, SVM and Gaussian Process are used to perform the single-tweet based stress detection over the feature space of each stress category, respectively. Precision and recall are leveraged to evaluate the performance. With the comment/response acts selected within $\Delta t$ after the tweet posted, assume for each stress category $C_{i} \in \mathcal{C}_{\text {ategory }}$, $T P\left(C_{i}, L v_{i}, \Delta t\right), T N\left(C_{i}, L v_{i}, \Delta t\right), F P\left(C_{i}, L v_{i}, \Delta t\right), F N\left(C_{i}, L v_{i}, \Delta t\right)$ represents the number of true positive, true negative, false positive and false negative samples detected of stress level $L v_{i}$, respectively. Thus,

$$
\begin{aligned}
& \operatorname{Precision}\left(C_{i}, \Delta t\right)=\frac{1}{\left|\mathcal{L}_{\text {evel }}\right|} \sum_{L v_{j} \in \mathcal{L}_{\text {evel }}} \operatorname{precision}\left(C_{i}, L v_{j}, \Delta t\right) \\
& \operatorname{Recall}\left(C_{i}, \Delta t\right)=\frac{1}{\left|\mathcal{L}_{\text {evel }}\right|} \sum_{L v_{j} \in \mathcal{L}_{\text {evel }}} \operatorname{recall}\left(C_{i}, L v_{j}, \Delta t\right) \\
& \text { where } \operatorname{precision}\left(C_{i}, L v_{j}, \Delta t\right)=\frac{T P\left(C_{i}, L v_{j}, \Delta t\right)}{T P\left(C_{i}, L v_{j}, \Delta t\right)+F P\left(C_{i}, L v_{i}, \Delta t\right)}, \text { and } \operatorname{recall}\left(C_{i}, L v_{j}, \Delta t\right) \\
&=\frac{T P\left(C_{i}, L v_{j}, \Delta t\right)}{T P\left(C_{i}, L v_{j}, \Delta t\right)+F N\left(C_{i}, L v_{j}, \Delta t\right)} \\
& \frac{{ }^{1} \text { One of the biggest Chinese micro-blog platform, http://t.qq.com/ }}{}
\end{aligned}
$$

\footnotetext{
${ }^{1}$ One of the biggest Chinese micro-blog platform, http://t.qq.com/
} 
Table 2. General Performance $(\Delta t=30$ minutes $)$

\begin{tabular}{|c|ccc|ccc|cccc|ccc|}
\hline \multirow{2}{*}{ Stress } & \multicolumn{3}{|c|}{ Naive Bayes } & \multicolumn{3}{c|}{ Logistic } & \multicolumn{3}{c|}{ SVM } & \multicolumn{3}{c|}{ Gaussian } \\
& Prec. & Rec. & F-ms. & Prec. Rec. F-ms. & Prec. Rec. & F-ms. & Prec. Rec. F-ms. \\
\hline Aca & 0.69 & 0.69 & 0.69 & 0.71 & 0.67 & 0.69 & 0.73 & 0.67 & 0.70 & 0.77 & 0.70 & 0.73 \\
\hline Aff & 0.53 & 0.72 & 0.61 & 0.81 & 0.72 & 0.76 & 0.87 & 0.81 & 0.83 & 0.44 & 0.38 & 0.41 \\
\hline Inter & 0.67 & 0.70 & 0.68 & 0.81 & 0.72 & 0.76 & 0.73 & 0.63 & 0.67 & 0.56 & 0.36 & 0.44 \\
\hline Self & 0.60 & 0.66 & 0.53 & 0.69 & 0.67 & 0.68 & 0.66 & 0.64 & 0.65 & 0.32 & 0.33 & 0.33 \\
\hline Avg. & $\mathbf{0 . 6 2}$ & $\mathbf{0 . 6 9}$ & $\mathbf{0 . 6 5}$ & $\mathbf{0 . 7 6}$ & $\mathbf{0 . 7 0}$ & $\mathbf{0 . 7 2}$ & $\mathbf{0 . 7 5}$ & $\mathbf{0 . 6 9}$ & $\mathbf{0 . 7 1}$ & $\mathbf{0 . 5 2}$ & $\mathbf{0 . 4 4}$ & $\mathbf{0 . 4 8}$ \\
\hline
\end{tabular}

Table 2 compares the performance with $\Delta t=30$ minutes. Averagely speaking, Logistic and SVM work the best in the four classifiers for all the stress categories, with the average F-measure over $70 \%$, which is $10.7 \%$ better than NB, and $50 \%$ better than Gaussian.

\subsection{Investigation of Time Sensitivity}

We investigate the time sensitivity of comment/response acts with different $\Delta t$ value. Logistic is selected as the classifier in this experiment since it performs the best of the three. Different time gap $\Delta t(=10,20,30,40$, and $+\infty$ minutes $)$ are selected to extract comment/response features, where all the interaction acts under a tweet are involved when $\Delta t=+\infty$. Fig. 1 shows the performance of different time sensitivity $(\Delta t)$. Obviously, for each stress category, the detection results are time-sensitive. With the increase of $\Delta t$, the performance first improves and then falls again as $\Delta t$ getting bigger. Particularly, when $\Delta t=30 \mathrm{~min}$, the detection performance reaches a peak, with the average $\mathrm{F}$-measure over $70 \%$. The result coincides with our daily observation. When a user posts a stressful tweet, his/her friends often comments in time to express care, comfort and encourage. With $\Delta t$ increases within $[0,30]$ (counted in minute), such effective information accumulates and makes the detection more accurate.

\subsection{Impact of Comment/Response Acts}

Table 3 compares the detection performance with/without comment/response acts under Logistic classifier. We set $\Delta t=30$ minutes for time-sensitive interaction acts. Due to user's indirect expression, without interaction acts cannot provide enough information and performs worse. When involving the comment/response acts, the average F-measure for all the stress categories reaches over $70 \%$, outperforms the other case $23.5 \%$, which proves the significance of timesensitive comment/response acts in stress detection.

\section{Conclusion}

In this paper, together with content of tweets, we involve time-sensitive comment/response acts under a tweet to help better detect stress revealed from a 


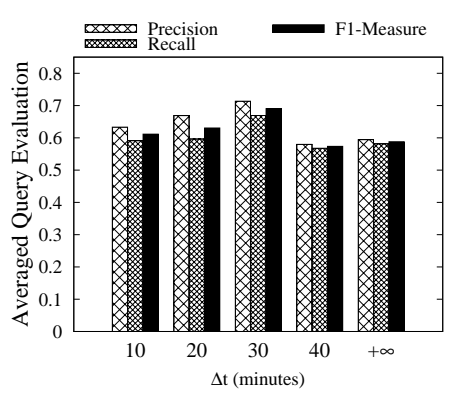

(a) Academic Stress

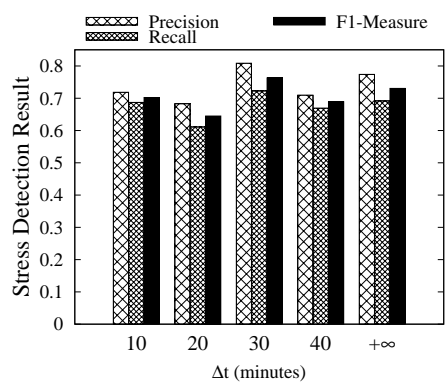

(c) Interpersonal Stress

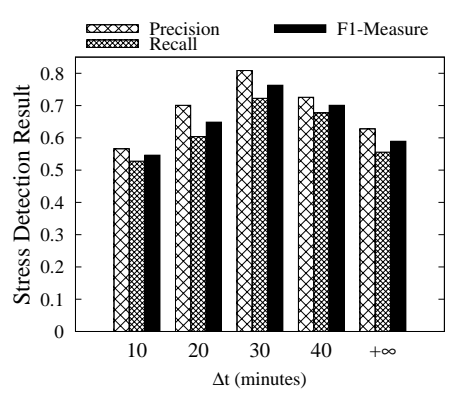

(b) Affection Stress

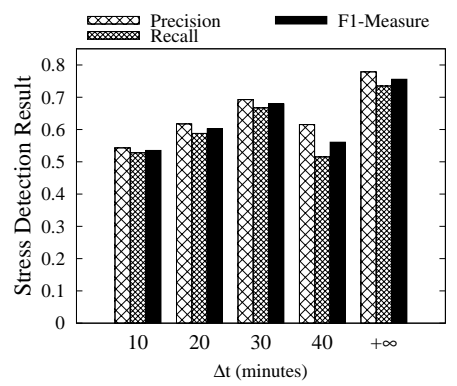

(d) Self-cognition Stress

Fig. 1. Performance with different time sensitivity $(\Delta t)$

Table 3. Comparison with/without comment/response acts

\begin{tabular}{|c|ccc|ccc|}
\hline \multirow{2}{*}{ Stress } & \multicolumn{3}{|c|}{ Without C/R acts } & \multicolumn{3}{c|}{ With C/R acts } \\
\cline { 2 - 7 } & Prec. & Rec. & F-meas. & Prec. & Rec. & F-meas. \\
\hline academic & 0.596 & 0.553 & 0.574 & 0.713 & 0.669 & 0.690 \\
\hline affection & 0.558 & 0.579 & 0.568 & 0.808 & 0.722 & 0.763 \\
\hline interpersonal & 0.744 & 0.613 & 0.669 & 0.808 & 0.722 & 0.763 \\
\hline self-cognition & 0.553 & 0.5 & 0.568 & 0.693 & 0.667 & 0.679 \\
\hline avg. & $\mathbf{0 . 6 1 3}$ & $\mathbf{0 . 5 6 1}$ & $\mathbf{0 . 5 8 6}$ & $\mathbf{0 . 7 5 6}$ & $\mathbf{0 . 6 9 5}$ & $\mathbf{0 . 7 2 4}$ \\
\hline
\end{tabular}

teenager's tweet. Focusing on the four main categories of adolescent stress (academic, affection, interpersonal, and self-cognition), we specify content features for each stress category, and involve several novel features extracted from timesensitive comment/response acts. 33 high school students aged 17 participated in our user study to evaluate the framework. Experimental results show that stress detection performance varies with different time sensitivity, and involving comment/response acts can better improve the F-measure of multi-category stress detection by $23.5 \%$. 


\section{Acknowledgement}

The work is supported by National Natural Science Foundation of China (61373022, 61370023, 61073004), and Chinese Major State Basic Research Development 973 Program (2011CB302203-2).

\section{References}

1. The week news. http://theweek.com/article/index/252199/the-rise-of-youthsuicide-in-china.

2. Sohu news. http://learning.sohu.com/s2012/shoot/, 2012.

3. Sohu news. http://learning/sohu.com/20130402/n371458123.shtml, 2013.

4. USA today. http://www.usatoday.com/story/news/nation/2014/02/11/stressteens- psychological/5266739, 2014.

5. Social media and the cost of caring. http://www, pewinternet.org/files/2015/01/PI_ Social-media-and-stress_0115151.pdf, 2015.

6. W. Che, Z. Li, Y. Guo, B. Qin, and T. Liu. Multilingual dependency-based syntactic and semantic parsing. In Proc. of CoNLL, pages 49-54, 2009.

7. W. Che, Z. Li, and T. Liu. LTP: a chinese language technology platform. In Proc. of Coling, pages 13-16, 2010.

8. M. Choudhury, S. Counts, and E. Horvitz. Social media as a measurement tool of depression in populations. In Proc. of ACM Web Science, pages 47-56, 2013.

9. M. Choudhury, M. Gamon, S. Counts, and E. Horvitz. Prediction depression via social media. In Proc. of ICWSM, 2013.

10. Q. Li, Y. Xue, J. Jia, and L. Feng. Helping teenagers relieve psychological pressures: a micro-blog based system. In Proc. of EDBT, pages 660-663, 2014.

11. H. Lin, J. Jia, Q. Guo, Y. Xue, J. Huang, L. Cai, and L. Feng. Psychological stress detection from cross-media microblog data using deep sparse neural nework. In Proc. of ICME, pages 1-6, 2014.

12. H. Lin, J. Jia, Q. Guo, Y. Xue, Q. Li, J. Huang, L. Cai, and L. Feng. User-level psychological stress detection from social media using deep neural network. In Proc. of MM, pages 507-516, 2014.

13. M. Park, D. McDonald, and M. Cha. Perception differences between the depressed and non-depressed users in twitter. In Proc. of ICWSM, 2013.

14. C. Tan, L. Lee, J. Tang, L. Jiang, M. Zhou, and P. Li. User-level sentiment analysis incorporating social networks. In Proc. of SIGKDD, pages 1397-1405, 2011.

15. X. Wang, C. Zhang, Y. Ji, L. Sun, and L. Wu. A depression detection model based on sentiment analysis in micro-blog social network. In Proc. of PAKDD, 2013.

16. Y. Xue, Q. Li, L. Jin, L. Feng, D. Clifton, and G. Clifford. Detecting adolescent psychological pressures from micro-blog. In Proc. of HIS, pages 83-94, 2014.

17. Y. Yang, J. Jia, S. Zhang, B. Wu, Q. Chen, J. Li, C. Xing, and J. Tang. How do your friends on social media disclose your emotions. In Proc. of AAAI.

18. Y.Shen, T. Kuo, I. Yeh, T. Chen, and S. Lin. Exploiting temporal information in a two-stage classification framework for content-based depression detection. In Proc. of PAKDD, pages 276-288, 2013.

19. Y. Zhang, J. Tang, J. Sun, Y. Chen, and J. Rao. Moodcast: emotion prediction via dynamic continuous factor graph model. In Proc. of ICDM.

20. J. Zhao, L. Dong, J. Wu, and K. Xu. Moodlens: an emoticon-based sentiment analysis system for chinese tweets. In Proc. of SIGKDD, pages 1528-1531, 2012. 